\title{
Maximum-mean uncertain measure
}

\author{
Xiang Li
}

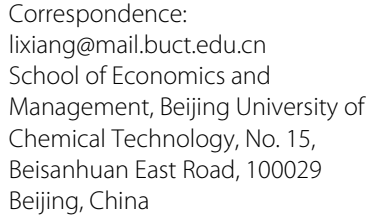

\begin{abstract}
Uncertain measure, which is used to indicate the degree of belief that an uncertain event may occur, is a set function satisfying the normality, duality, and subadditivity axioms. Although the tools of uncertain measure, uncertainty space, and uncertain variable have been well developed and became the foundations of uncertainty theory, there is no study on how to construct the uncertainty space for an uncertain variable, which seriously hinders the applications of uncertainty theory in practice. In this paper, we propose an expert knowledge-based construction approach for uncertainty space with maximum-mean uncertain measure, which takes the maximum and mean operations on confidence function.
\end{abstract}

Keywords: Maximum-mean uncertain measure; Confidence function; Expert knowledge

\section{Introduction}

Uncertainty theory introduced by Liu [1] is a branch of nonadditive measure theory for describing human uncertainty. Compared with randomness, Liu's uncertainty amounts to subject indeterminacy arising from information loss on some exact value of a certain quantity. The main difference between randomness and Liu's uncertainty is that the former is used to model unfixed values, while the latter is used to model fixed but ill-known values. For example, if we toss a coin in the air and choose between head and tail before it lands on the ground, the outcome is random since both head and tail are possible. However, if we make the choice after the coin has landed on the ground, the outcome is uncertain since it has been fixed although we do not know the result.

Nowadays, uncertainty theory has become a branch of axiomatic mathematics for modeling human uncertainty, and it has been well developed and applied to uncertain programming [2], uncertain statistics [3], uncertain risk analysis [4], uncertain set [5], uncertain logic [6], uncertain inference [7,8], uncertain process [9], uncertain renewal process [10], uncertain calculus [11], uncertain differential equation [12], uncertain finance [13], and so on. The latest results related to uncertainty theory may be found in [14].

Uncertain measure and uncertain variable are two basic concepts in uncertainty theory. Uncertain measure, which is used to indicate the degree of belief that an uncertain event may occur, was defined in [1] as a set function satisfying the normality, duality, and subadditivity axioms. The normality is used to ensure that degree of belief is consistent with human thinking, and the duality is an extension of the law of contradiction. For a

(c) 2015 Li; licensee Springer. This is an Open Access article distributed under the terms of the Creative Commons Attribution License (http://creativecommons.org/licenses/by/4.0), which permits unrestricted use, distribution, and reproduction in any medium, provided the original work is properly credited. 
sequence of countable events, subadditivity means that the entire degree of belief must be less than or equal to the sum of their individual degrees of belief. Liu [15] proved the monotonicity theorem, null-additivity theorem, and asymptotic theorem, and Gao [16] studied some properties of continuous uncertain measures. The product uncertain measure of compound event was defined by Liu [17] as the minimum of uncertain measures of individual events, and the product uncertain measure theorem was proved by Peng and Iwamura [18]. In order to study the dependence of uncertain events, Liu [1] defined the conditional uncertain measure of an event given that some other event has occurred. Uncertain variable is used to represent quantity with uncertainty, which was defined in [1] as a measurable function from uncertainty space to the set of real numbers.

Possibility theory is another type of nonadditive measure theory for dealing with the subjective uncertainty. Possibility measure was defined by Zadeh [19] as a set function satisfying the normality, nonnegativity, and maximality. Necessity measure was also defined by Zadeh [20] as the dual part of possibility measure. It is proved that both possibility measure and necessity measure satisfy the properties of normality, nonnegativity, and monotonicity. However, neither of them are self-dual. Since the duality is intuitive and important in real problems, Liu and Liu [21] defined a credibility measure as the average of possibility measure and necessity measure, which was lately proved to be a set function satisfying normality, monotonicity, duality, and partial maximality [22]. As an extension of credibility measure, Liu [1] proposed the uncertain measure by slacking the partial maximality to subadditivity, which generalizes the uncertain model to describe ill-known but fixed value.

Although the tools of uncertain measure, uncertainty space, and uncertain variable have been well developed and became the foundations for uncertainty theory, there is no study on how to construct the uncertainty space for an uncertain variable. This seriously hinders the applications of uncertainty theory in practice. The purpose of this paper is to provide a general construction approach for uncertainty space with maximum-mean uncertain measure based on the expert knowledge. The rest of this paper is organized as follows. The 'Preliminaries' section reviews some basic definitions and properties on uncertainty theory. The 'Confidence function' section introduces a concept of confidence function. In the 'Maximum-mean uncertain measure' section, we propose the concept of maximummean uncertain measure based on the maximum and mean operations on confidence function, and we provide a general procedure on generating an uncertainty space with the maximum-mean uncertain measure. The 'An illustrative example' section provides a practical example to illustrate how to construct an uncertainty space for an uncertain quantity. Finally, a brief summary is given.

\section{Preliminaries}

This section will briefly review some basic concepts and properties of uncertainty theory. Let $\Gamma$ be a nonempty set, and let $\Lambda$ be a $\sigma$-algebra over $\Gamma$. Each element of $\Lambda$ is called an event. In order to present an axiomatic definition of uncertain measure, it is necessary to assign to each event a number which indicates the degree of belief that the event will occur. In order to ensure that this assignment has certain meaningful mathematical properties, Liu [1] proposed the following three axioms:

Axiom 1. (Normality) $\mathrm{M}\{\Gamma\}=1$; 
Axiom 2. (Duality) $\mathrm{M}\{A\}+\mathrm{M}\left\{A^{c}\right\}=1$ for any $A \in \Lambda$;

Axiom 3. (Subadditivity) For every countable sequence of events $\left\{A_{i}\right\}$, we have:

$$
\mathrm{M}\left\{\bigcup_{i=1}^{\infty} A_{i}\right\} \leq \sum_{i=1}^{\infty} \mathrm{M}\left\{A_{i}\right\} .
$$

The normality is used to ensure that degree of belief is consistent with human thinking. The duality is an extension of the law of contradiction, which ensures that an event and its complement cannot occur simultaneously. For a sequence of countable events, subadditivity means that the entire degree of belief must be less than or equal to the sum of their individual degrees of belief.

Definition 2.1. Let $\Gamma$ be a nonempty set, and let $\Lambda$ be a $\sigma$-algebra (Liu [1]). The set function $M$ is called an uncertain measure if it satisfies the normality, duality, and subadditivity axioms.

Liu [15] proved the monotonicity theorem for uncertain measure. That is, for any events $A_{1} \subseteq A_{2}$, we have $\mathrm{M}\left\{A_{1}\right\} \leq \mathrm{M}\left\{A_{2}\right\}$. In addition, the null-additivity theorem and asymptotic theorem were also proved. Liu [17] defined the product uncertain measure for compound event, and Liu [1] defined a conditional uncertain measure of an event after it has been learned that some other event has occurred.

Definition 2.2. Suppose that $\Gamma$ is a nonempty set, $\Lambda$ is a $\sigma$-algebra, and $M$ is an uncertain measure (Liu [1]). Then, the triplet $(\Gamma, \Lambda, M)$ is called an uncertainty space.

Definition 2.3. An uncertain variable $\xi$ is a measurable function from an uncertainty space $(\Gamma, \Lambda, M)$ to the set of real numbers, that is, for any Borel set $B$ of $\Re$, we have (Liu $[1])$ :

$$
\{\gamma \in \Gamma \mid \xi(\gamma) \in B\} \in \Lambda
$$

Example 2.1. Suppose that $\Gamma$ is a nonempty set containing three points $\gamma_{1}, \gamma_{2}$, and $\gamma_{3}$. Let $\Lambda$ be the power set of $\Gamma$. Define $M\{\emptyset\}=0, M\left\{\gamma_{1}\right\}=0.2, M\left\{\gamma_{2}\right\}=0.4, M\left\{\gamma_{3}\right\}=0.6$, $\mathrm{M}\left\{\gamma_{1}, \gamma_{2}\right\}=0.4, \mathrm{M}\left\{\gamma_{2}, \gamma_{3}\right\}=0.8, \mathrm{M}\left\{\gamma_{1}, \gamma_{3}\right\}=0.6$ and $\mathrm{M}\{\Gamma\}=1$. It is easy to prove that $M$ satisfies the normality, duality, and subadditivity axioms. Then, $M$ is an uncertain measure, and the triplet $(\Gamma, \Lambda, M)$ is an uncertainty space. Define a real function $\xi\left(\gamma_{1}\right)=$ $x_{1}, \xi\left(\gamma_{2}\right)=x_{2}$ and $\xi\left(\gamma_{3}\right)=x_{3}$. Since $\Lambda$ is the largest $\sigma$-algebra over $\Gamma$, for any Borel set $B$ of $\Re$, it is clear that $\{\xi \in B\} \in \Lambda$. Thus, $\xi$ is a measurable function, that is, an uncertain variable, on uncertainty space $(\Gamma, \Lambda, M)$.

\section{Confidence function}

Let $\xi$ be an uncertain variable. For theoretical studies, we always assume that we can define an uncertainty space $(\Gamma, \Lambda, M)$ with known uncertain measure such that $\xi$ is a measurable function from this space to the set of real numbers. However, it is difficult to construct an uncertainty space in practice since there is no efficient construction approach for uncertain measure.

If $\Lambda$ is finite and has a small number of elements, we may define an uncertain measure by enumerating the degree of beliefs for all events according to the expert knowledge. 
However, if the cardinality of $\Lambda$ is infinite, it is impossible to do in this way since the number of events is unmanageable. Therefore, an efficient construction approach for uncertain measure should be necessary. This paper defines a maximum-mean uncertain measure and proposes a construction approach based on the maximum and mean operations on confidence function. First, we introduce the concept of confidence function.

Let $\Gamma=\left\{x_{1}, x_{2}, \cdots\right\}$ be the universal set, i.e., the collection of candidates. Suppose that there are $m$ experts with confidences $\left\{v_{1}, v_{2}, \cdots, v_{m}\right\}$, which are given by an expert of experts. Without loss of generality, we assume that each candidate associated with one and only one confidence under the following rules: (i) if some candidate receives no support from any expert, we assign it with confidence 0 ; (ii) if some candidate receives support from one and only one expert, for example, expert $i$, we assign $v_{i}$ to it; (iii) if some candidate receives supports from more experts, for example, experts $i_{1}, i_{2}, \cdots, i_{m_{i}}$, we assign it with the confidence $\max \left\{v_{i_{1}}, v_{i_{2}}, \cdots, v_{i_{m_{i}}}\right\}$.

A confidence function $\mu$ is a mapping from $\Gamma$ to $[0,1]$ such that $\mu\left(x_{i}\right)$ denotes the confidence on the $i$ th candidate $x_{i}$, which has the following form:

$$
\mu(x)=\left\{\begin{array}{ccc}
\mu_{1}, & \text { if } x=x_{1} \\
\mu_{2}, & \text { if } x=x_{2} \\
\vdots & \vdots \\
\mu_{n}, & \text { if } x=x_{n} .
\end{array}\right.
$$

The confidence function indicates a relative preference among different candidates for the uncertain quantity. Generally speaking, we prefer to take the candidate that has the largest confidence value.

\section{Maximum-mean uncertain measure}

This section defines a maximum-mean uncertain measure based on the confidence function, which expresses the degree of belief that an uncertain event will occur.

First, let us consider the confidence on a set of candidates. As we know, each uncertain quantity has one and only one correct value. In other words, there is only one expert providing the correct estimation, and the others all provide the wrong estimation. The uncertainty arises from the difficulty that we do not know who is the correct expert. Therefore, if we believe that the uncertain quantity takes a value belonging to set $A$, the confidence should be the maximum value among all confidences on candidates in set $A$.

Example 4.1. Suppose that $\xi$ is an uncertain quantity with confidence function:

$$
\mu(x)=\left\{\begin{array}{l}
0.3, \text { if } x=10 \\
0.5, \text { if } x=15 \\
0.7, \text { if } x=20 \\
1.0, \text { if } x=30
\end{array}\right.
$$

Since candidate 30 has the maximum confidence, we prefer to believe that $\xi$ will take value 30 . On the other hand, for the uncertain event $\{\xi \neq 30\}$, the confidence should be the maximum confidence on candidates 10,15 , and 20, that is, $0.3 \vee 0.5 \vee 0.7=0.7$. 
Let $\xi$ be an uncertain quantity with confidence function $\mu$. Based on the above analysis, for each Borel set $A$, we calculate the confidence of the uncertain event $\{\xi \in A\}$ by:

$$
\mu_{A}=\max _{x_{i} \in A} \mu_{i}
$$

and calculate the confidence of its complement $\left\{\xi \in A^{c}\right\}$ by:

$$
\mu_{A^{c}}=\max _{x_{i} \in A^{c}} \mu_{i}
$$

If $\mu_{A}>0$, we believe that it is possible that $\xi$ takes a value in set $A$, and the degree of belief should have a strong positive correlation with respect to the confidence such that a larger confidence leads to a larger degree of belief.

Theorem 4.1. Suppose that $\Gamma$ is a nonempty set $\left\{x_{1}, x_{2}, \cdots\right\}$, and $\mu$ is a nonnegative real function satisfying:

$$
\max _{x_{i} \in \Gamma} \mu_{i}>0 .
$$

Let $\Lambda$ be a $\sigma$-algebra over $\Gamma$. For each $A \in \Lambda$, we define:

$$
\mathrm{M}\{A\}=\frac{\max _{x_{i} \in A} \mu_{i}}{\max _{x_{i} \in A} \mu_{i}+\max _{x_{i} \in A^{c}} \mu_{i}} .
$$

Then, the set function $M$ is an uncertain measure (called the maximum-mean uncertain measure), and the triplet $(\Gamma, \Lambda, M)$ is an uncertainty space.

Proof: We prove that the set function $M$ satisfies the normality axiom, duality axiom, and subadditivity axiom. First, according to condition (5), the normality can be obtained immediately from formula (6) by showing:

$$
\max _{x_{i} \in \Gamma^{c}} \mu_{i}=\max _{x_{i} \in \emptyset} \mu_{i}=0 .
$$

Furthermore, let us prove the duality. For any $A \in \Lambda$, it follows from (6) that:

$$
\mathrm{M}\{A\}=\frac{\max _{x_{i} \in A} \mu_{i}}{\max _{x_{i} \in A} \mu_{i}+\max _{x_{i} \in A^{c}} \mu_{i}}, \mathrm{M}\left\{A^{c}\right\}=\frac{\max _{x_{i} \in A^{c}} \mu_{i}}{\max _{x_{i} \in A^{c}} \mu_{i}+\max _{x_{i} \in A} \mu_{i}},
$$

which implies that $\mathrm{M}\{A\}+\mathrm{M}\left\{A^{c}\right\}=1$. Finally, we prove the subadditivity. For any events $A, B \in \Lambda$, without loss of generality, we assume that:

$$
\max _{x_{i} \in A} \mu_{i} \leq \max _{x_{i} \in B} \mu_{i}
$$

Then, it can be proved that:

$$
\max _{x_{i} \in A \cup B} \mu_{i}=\max _{x_{i} \in A} \mu_{i} \vee \max _{x_{i} \in B} \mu_{i}=\max _{x_{i} \in B} \mu_{i} .
$$

The argument breaks down into two cases. If:

$$
\max _{x_{i} \in A \cap B^{c}} \mu_{i}<\max _{x_{i} \in A^{c} \cap B^{c}} \mu_{i},
$$

it follows from $B^{c}=\left(A \cap B^{c}\right) \cup\left(A^{c} \cap B^{c}\right)$ that:

$$
\max _{x_{i} \in B^{c}} \mu_{i}=\max _{x_{i} \in A^{c} \cap B^{c}} \mu_{i} .
$$


Then, Equations (7) and (8) prove that:

$$
\frac{\max _{x_{i} \in A \cup B} \mu_{i}}{\max _{x_{i} \in A \cup B} \mu_{i}+\max _{x_{i} \in A^{c} \cap B^{c}} \mu_{i}}=\frac{\max _{x_{i} \in B} \mu_{i}}{\max _{x_{i} \in B} \mu_{i}+\max _{x_{i} \in B^{c}} \mu_{i}},
$$

which implies that $\mathrm{M}\{A \cup B\}=\mathrm{M}\{B\} \leq \mathrm{M}\{A\}+\mathrm{M}\{B\}$. Otherwise, we have:

$$
\max _{x_{i} \in A \cap B^{c}} \mu_{i} \geq \max _{x_{i} \in A^{c} \cap B^{c}} \mu_{i}
$$

In this case, it can be proved that:

$$
\max _{x_{i} \in B^{c}} \mu_{i}=\max _{x_{i} \in A \cap B^{c}} \mu_{i} \leq \max _{x_{i} \in A} \mu_{i} .
$$

It follows from $A^{c}=\left(A^{c} \cap B\right) \cup\left(A^{c} \cap B^{c}\right)$ that:

$$
\begin{aligned}
\max _{x_{i} \in A^{c}} \mu_{i} & =\max _{x_{i} \in A^{c} \cap B^{c}} \mu_{i} \vee \max _{x_{i} \in A^{c} \cap B} \mu_{i} \\
& \leq \max _{x_{i} \in A \cap B^{c}} \mu_{i} \vee \max _{x_{i} \in A^{c} \cap B} \mu_{i} \\
& \leq \max _{x_{i} \in A} \mu_{i} \vee \max _{x_{i} \in B} \mu_{i} \\
& =\max _{x_{i} \in B} \mu_{i} .
\end{aligned}
$$

According to inequalities (9) and (10), we have:

$$
\mathrm{M}\{A\} \geq \frac{\max _{x \in A} \mu(x)}{\max _{x \in A} \mu(x)+\max _{x \in B} \mu(x)}, \mathrm{M}\{B\} \geq \frac{\max _{x \in B} \mu(x)}{\max _{x \in A} \mu(x)+\max _{x \in B} \mu(x)}
$$

which implies that $\mathrm{M}\{A\}+\mathrm{M}\{B\} \geq 1 \geq \mathrm{M}\{A \cup B\}$. The subadditivity is proved. The proof is complete.

Remark 4.1. As a special uncertain measure, the maximum-mean uncertain measure also satisfies the monotonicity theorem, null-additivity theorem, and asymptotic theorem.

Let $\xi$ be an uncertain quantity with confidence function $\mu$. Define an uncertainty space ( $\Gamma, \Lambda, M)$ according to Theorem 4.1. Then, $\xi$ can be well described as $\xi\left(x_{i}\right)=x_{i}, \forall x_{i} \in \Gamma$. A general procedure on generating an uncertainty space with maximum-mean uncertain measure for $\xi$ is summarized as follows:

Step 1. Define the set of candidates $\Gamma=\left\{x_{1}, x_{2}, \cdots\right\}$.

Step 2. Define a confidence function $\mu: \Gamma \rightarrow[0, \infty)$.

Step 3. Take a $\sigma$-algebra $\Lambda$ over $\Gamma$.

Step 4. Define a maximum-mean uncertain measure $M$ according to formula (6).

Step 5. Define $\xi\left(x_{i}\right)=x_{i}$ for all $x_{i} \in \Gamma$.

Remark 4.2. If there is a candidate with confidence zero, for example, $\mu_{1}=0$, then, we have $\mathrm{M}\left\{\xi=x_{1}\right\}=0$. If all candidates have the same confidence, then, the degree of belief that $\xi$ takes value $x_{i}$ is $\mathrm{M}\left\{\xi=x_{i}\right\}=0.5$ for each $1 \leq i \leq n$.

\section{An illustrative example}

This practical example illustrates how to construct an uncertainty space for an uncertain quantity. Yufu Ning is a middle-aged college professor. He is my friend, but I 
have no idea on the exact value of his age, except that it should take value in $\Gamma=$ $\{41,42,43,44,45,46,47,48,49,50\}$. In order to obtain a better understanding on it, I send a questionnaire to ten of our common friends. The first question is "How old is Prof. Ning," and the second question is "What are the degrees of belief that Prof. Ning is less than or equal to 45 and Prof. Ning is larger than 45." The received answers are summarized by Table 1, where the third column shows the candidates selected by these experts, the fourth column shows the confidences of experts, the fifth column shows the degrees of belief that Prof. Ning is less than or equal to 45, and the last column shows the degrees of belief that Prof. Ning is larger than 45. Here, the confidences of experts are given by Prof. Ning.

It follows from Table 1 that the confidence function is $\mu(41)=0.4, \mu(44)=0.8$, $\mu(45)=0.8, \mu(46)=0.9$, and $\mu(47)=0.9$. Take $\Lambda$ to be the power set of $\Gamma$. According to formula (6), we can define a maximum-mean uncertain measure $M$. Then, Ning's age may be treated as the identity uncertain variable $\xi$ on uncertainty space $(\Gamma, \Lambda, M)$.

Now, we consider the maximum-mean uncertain measure of events $\{\xi \leq 45\}$ and $\{\xi>$ $45\}$. According to formula (6), it is easy to calculate that $\mathrm{M}\{\xi \leq 45\}=0.8 /(0.8+0.9)=$ 0.471 and $\mathrm{M}\{\xi>45\}=0.9 /(0.9+0.8)=0.529$. On the other hand, according to Table 1 , we aggregate the experts' knowledge as follows:

- Prof. Ning is less than or equal to 45 with:

$$
\frac{\sum_{i=1}^{10} v_{i} \alpha_{i}}{\sum_{i=1}^{10} v_{i} \alpha_{i}+\sum_{i=1}^{10} v_{i} \beta_{i}}=0.472 .
$$

- Prof. Ning is larger than 45 with:

$$
\frac{\sum_{i=1}^{10} v_{i} \beta_{i}}{\sum_{i=1}^{10} v_{i} \alpha_{i}+\sum_{i=1}^{10} v_{i} \beta_{i}}=0.528 .
$$

Therefore, the maximum-mean uncertain measure satisfies the experts' knowledge.

\section{Conclusion}

The main contribution of this paper is the constructive approach of an uncertainty space with maximum-mean uncertain measure for an uncertain variable. It is the first

Table 1 Answers on Prof. Ning's age

\begin{tabular}{clcccc}
\hline & Expert & Candidate & Confidence $\left(\boldsymbol{v}_{\boldsymbol{i}}\right)$ & $\mathbf{\leq 4 5}\left(\boldsymbol{\alpha}_{\boldsymbol{i}}\right)$ & $\mathbf{>} \mathbf{4 5}\left(\boldsymbol{\beta}_{\boldsymbol{i}}\right)$ \\
\hline 1 & Dr. Chen & 47 & 0.7 & 0.4 & 0.6 \\
2 & Dr. Gao & 41 & 0.4 & 0.7 & 0.2 \\
3 & Prof. Ke & 45 & 0.6 & 0.8 & 0.6 \\
4 & Dr. Li & 45 & 0.8 & 0.5 & 0.4 \\
5 & Prof. Qin & 47 & 0.9 & 0.5 & 0.5 \\
6 & Dr. Wen & 45 & 0.4 & 0.3 & 0.7 \\
7 & Prof. Zhou & 45 & 0.3 & 0.9 & 0.1 \\
8 & Prof. Zhang & 46 & 0.9 & 0.0 & 1.0 \\
9 & Prof. Li & 44 & 0.8 & 0.6 & 0.4 \\
10 & Prof. Zhu & 45 & 0.7 & 0.8 & 0.8 \\
\hline
\end{tabular}


such study on how to characterize a practical uncertain quantity mathematically based on expert knowledge, which makes it possible to apply uncertainty theory to real-life problems.

\section{Acknowledgements}

This work was supported by the Specialized Research Fund for the Doctoral Program of Higher Education of China (No. 20110009120036).

Received: 14 November 2014 Accepted: 1 January 2015

Published online: 12 February 2015

\section{References}

1. Liu, B: Uncertainty theory. 2nd ed. Springer-Verlag, Berlin, Germany (2007)

2. Liu, B: Theory and practice of uncertain programming. 2nd ed. Springer-Verlag, Berlin, Germany (2009)

3. Wang, XS, Gao, ZC, Guo, HY: Uncertain hypothesis testing for two experts' empirical data. Math. Comput. Model. 55, 1478-1482 (2012)

4. Peng, J: Risk metrics of loss function for uncertain system. Fuzzy Optim. Decis. Making. 12(1), 53-64 (2013)

5. Liu, B: Membership functions and operational law of uncertain sets. Fuzzy Optim. Decis. Making. 11(4), 387-410 (2012)

6. Li, X, Liu, B: Hybrid logic and uncertain logic. J. Uncertain Syst. 3(2), 83-94 (2009)

7. Gao, X, Gao, Y, Ralescu, DA: On Liu's inference rule for uncertain systems. Int. J. Uncertain. Fuzz. 18(1), 1-11 (2010)

8. Liu, B: Uncertain set theory and uncertain inference rule with application to uncertain control. J. Uncertain Syst. 4(2), 83-98 (2010)

9. Liu, B: Fuzzy process, hybrid process and uncertain process. J. Uncertain Syst. 2(1), 3-16 (2008)

10. Yao, K, Li, X: Uncertain alternating renewal process and its application. IEEE Trans. Fuzzy Syst. 20(6), 1154-1160 (2012)

11. Yao, K: Uncertain calculus with renewal process. Fuzzy Optim. Decis. Making. 11(3), 285-297 (2012)

12. Barbacioru, IC: Uncertainty functional differential equations for finance. Surveys Math. Appl. 5, 275-284 (2010)

13. Liu, B: Toward uncertain finance theory. J. Uncertain. Anal. Appl. 1(1) (2013)

14. Liu, B: Uncertainty theory. 4th ed. [Online] Available: http://orsc.edu.cn/liu/ut.pdf

15. Liu, B: Uncertainty theory: a branch of mathematics for modeling human uncertainty. Springer-Verlag, Berlin, Germany (2010)

16. Gao, X: Some properties of continuous uncertain measure. Int. J. Uncertain. Fuzz. 17(3), 419-426 (2009)

17. Liu, B: Some research problems in uncertainty theory. J. Uncertain Syst. 3(1), 3-10 (2009)

18. Peng, ZX, Iwamura, K: Some properties of product uncertain measure. J. Uncertain Syst. 6(4), 263-269 (2012)

19. Zadeh, LA: Fuzzy sets as a basis for a theory of possibility. Fuzzy Set. Syst. 1(1), 3-28 (1978)

20. Zadeh, LA: Mathematical Frontiers of the Social and Policy Sciences. In: Hayes et al. (ed.), pp. 69-129. Westview Press, Boulder, Cororado, (1979)

21. Liu, B, Liu, Y: Expected value of fuzzy variable and fuzzy expected value models. IEEE Trans. Fuzzy Syst. 10(4), 445-450 (2002)

22. Li, X, Liu, B: A sufficient and necessary condition for credibility measures. Int. J. Uncertain. Fuzz. 14(5), $527-535$ (2006)

\section{Submit your manuscript to a SpringerOpen ${ }^{\circ}$ journal and benefit from:}

$\rightarrow$ Convenient online submission

Rigorous peer review

- Immediate publication on acceptance

- Open access: articles freely available online

- High visibility within the field

- Retaining the copyright to your article

Submit your next manuscript at $>$ springeropen.com 